\title{
Research of computer image aesthetics' classification and assessment based on support vector machine
}

\author{
Jian-liang Xiong ${ }^{1,}$, , Yan-mei Yang ${ }^{2, b}$ \\ ${ }^{1}$ Computer Engineering Department, Tangshan Vocational College of Science and Technology, \\ Tangshan, China \\ ${ }^{2}$ Computer Engineering Department, Tangshan Vocational College of Science and Technology, \\ Tangshan, China \\ axxxx9527@126.com, byangyanmei604@163.com
}

Keywords: Support vector machine, Computer image aesthetics; Classification, Evaluation.

\begin{abstract}
Computer image aesthetics is an interdisciplinary research field, which covering visual arts, psychology, information theory and other disciplines. And it depends on the image processing and computer vision to solve specific problems. This paper will design a comprehensive computer image aesthetic evaluation model, containing aesthetic categories and scores prediction, which will be realized by SVM classifier and SVR algorithm. Experiments show that the model's experimental results are in conformity with human aesthetic perception results.
\end{abstract}

\section{Introduction}

Cmputer image aesthetics is an interdisciplinary research field, which covering visual arts, psychology, information theory and other disciplines. So far, there are no unified applicable standard for computer image aesthetics, and evaluation are with strong subjectivity. So it is a very challenging problem to evaluate image aesthetics with computer. At present, there are many researchers putting forward thier own aesthetic evaluation model and solutions for different specific scope. Datta's ${ }^{[1]}$ aethetic evaluation model includes factors considered photography, which are color, composition and distribution. Wong et al. ${ }^{[2]}$ increase the consideration of the local regional characteristics. In addition, Machado $^{[3]}$ introduce the information theory into computer image aesthetics category, evaluating the pictorial image through analyzing image's order degree and harmonious degree.

Studying the results of other researchers and combining their typical characteristecs, this paper will build a computer image aesthetic evaluaiton model can be widely used in different types. This paper's image aethetic evaluaiton research is mainly composed of three parts, which are aesthetic feature extraction, aesthetic level classification, and aethetic score evaluation. The paper uses machine learning methods to establish the evaluation model. Among them, aesthetic image classification model is based on support vecotr machine, dividing the image to high and low aethetic. Score evaluation model uses the support vector machine to provide specific scores for imange beauty. These two models respectively correspond to the classification and regression problems fo machine learning. It realizes the machine's automatic classification, and provide a score close to people's aethetic habit.

\section{The Feature Extraction of Computer Image Aesthetics.}

Currently, the existing aesthetic evaluation algorithm mainly extracts the low-level visual features and high-level aesthetic features. Based on low-level features, this paper combines with the related theories of fine arts, psychology, and photography, looking for human perception and aesthetic' related characteristic, and expressing with appropriate mathematical way.

\subsection{Low-level visual feature.}

Low-level visual feature has intuitive visual expression significance. As the original data, it can objectively describe the image content. 
(1) Color feature

Color features reflect the overall characteristics of computer images, usually can be described by color histogram, color moment, other related vectors. Due to the color histogram and color moment characteristics can express the

Image color distribution features well, and their calculation is relatively simple, they are commonly used in image processing.

(2) Texture feature

Texture feature describes the spatial distribution pattern of pixel intensity, which is an important characteristic of a performance image. Image texture features describe color spatial uniformity and light distribution of images or small area. Texture feature with the characteristics of rotation invariance, having strong ability to resistant the noise, and reflecting the properties of the image itself, is one of the main characteristics of image processing and pattern recognition. In computer image aesthetic calculation, the phenomenon of image's uniformity, meticulous and roughness, is often a standard to evaluate the computer image beauty or unattractiveness. In general, the uniform, meticulous, and orderly images are more aesthetic.

(3) Shape feature

Shape feature plays an important role in the machine vision system. Computer image's edge shape information can cause the observer's attention, thus affect the observer's aesthetic judgment. Image shape feature are often expressed with contour features and regional characteristics. Contour feature consider the outer boundary information, and regional feature consider the shape region of entire image.

\subsection{The Laws of Aesthetics Characteristics.}

Since the low-level is relatively simple, some implicit relationship between the aesthetic feeling and characteristics of image can't be expressed intuitively. Early experiments also show that simple low-level features' experimental results are also not very ideal. Relative to the constructing aesthetic evaluation way through the bottom-up low-level features, some research put forward the method to using aesthetic principle to extract high-level aesthetic characteristics, obtaining a more ideal result. These characteristics are refined from the related theory of aesthetics, photography, and art. This article will summarize the existing research results, combining with the aesthetic psychology, photography rules and aesthetic measure associated with human aesthetic perception knowledge, to calculate a series of characteristics. The experimental verification shows that the high-level aesthetic characteristics can batter describe the computer image aesthetic information.

\subsection{Regional Characteristics.}

The existing visual psychology research shows that significant regional observer can attract the most attention, which is due to the significant region contains much useful information. Luo ${ }^{[4]}$ emphasized the importance of significant region in the computer image aesthetics evaluation. Wong et al. ${ }^{[2]}$ use the Itti visual attention system ${ }^{[5]}$ to detect significant region and extract the corresponding regional characteristics. Their results show the effectiveness of significant regional characteristics in the computer image aesthetic evaluation.

For the computer image's key areas, people often pay more attention to the regional distribution of color, texture, shape size, shape scale, etc ${ }^{[6]}$. Therefore, when considering the characteristics of computer image's key area, combining with the computer image characteristics, this article will calculate the HSV color space and shape proportion ${ }^{[7]}$. The color moment include $\mathrm{H}, \mathrm{S}, \mathrm{V}$ three moments of three channels. Shape ratio refers to the rate of the key regional pixels' total number to the original computer image's total number.

We will add the extracted key regional features to the global feature data for learning and training, to improve the performance of the classifier and regression model. 


\section{Construction of computer image aesthetic evaluation model based on support vector machine.}

This paper builds disaggregation model and score prediction model for computer image aesthetic evaluation research, each of the two models' result can be used as a supplement to the other side. The classification model is completed by support vector machine, and the score prediction model is complete by support vector machine regression algorithm.

\subsection{Support Vector Machine Algorithm}

Support vector machine is a widely applied classifier. It was first put forward by Vapnik, Cortes and their team in 1995. Through studying the input samples, the purpose of SVM is to determine a clear interval in the vector space, distinguishing the different samples. This demarcation line dividing the interval is support vector. In linear SVM, the support vector is a straight line, which can be determined by only a small amount of samples. Linear support vector classification function is defined as follows.

$$
f(x)=\langle w, x\rangle+b \quad \text { with } \quad w \in \chi, b \in R
$$

Where, $<\mathrm{W}, \mathrm{X}>$ expresses dot product between vectors. $\mathrm{X}$ expresses the vector space of input sample. The purpose of the function is to find a w, which can make the function minimum. The function can be transformed into the problem of solving the convex optimization.

$$
\begin{aligned}
& \min \theta=\frac{1}{2}\|w\|^{2} \\
& \text { s.t.c }\left(w \cdot x_{i}-b\right) \geq 1, \quad 1 \leq i \leq n
\end{aligned}
$$

Nonlinear SVM algorithm also uses kernel function mapping principle, whose basic idea is to map the nonlinear low dimension space to high dimension space, the essence of which is to transform the low-dimensional space to high-dimensional space data by linear transformation, solving the optimal hyper plane in the high-dimensional space. This method is easy to deal with nonlinear problem, and solve the problem trapped in local minima and dimension disaster. The Gaussian function nuclear is a method widely used in nonlinear SVM. After completing the feature extraction, normalize these characteristics. RBF is generally defined as follows.

$$
K\left(x, x^{\prime}\right)=\exp \left(-\gamma\left\|x-x^{\prime}\right\|_{2}^{2}\right)
$$

$\mathrm{x}$ and $\mathrm{x}$ ' express two samples' characteristics. $\left\|x-x^{\prime}\right\|_{2}^{2}$ expresses the Euclidean distance between the characteristics samples. As weights, $\gamma$ adjusts the importance of sample distance in RBF. In the SVM classifier, $\mathrm{C}$ is the penalty function to adjust sample effects on RBF. Finally, through the multi-parameter optimization, we can find the optimal $\gamma$ and $C$ value, thereby improving the performance of the SVM classifier.

\subsection{Support Vector Machine Regression Algorithm}

In order to evaluate the computer image aesthetics through computer aided, this paper adopted the SVR algorithm for computer image aesthetics scores forecasts. The basis ideas of SVR are similar to SVM classifier, and the difference is that the SVR is used to solve regression problem. In this paper, the SVR model is build by $\varepsilon$-SVR method. $\varepsilon$ represents the target deviation, which is the most deviation between objective function $\mathrm{f}(\mathrm{x})$ with the actual value in the sample training. In other words, when the error is less than $\varepsilon$, we still treat the results of objective function as right results. Similar to SVM, problems can be regarded as solving the convex optimization problem.

$$
f(x)=\langle w, x\rangle+b \quad \text { with } \quad w \in \chi, b \in R
$$


Similar to classification problem, we should also introduce a penalty factor for objective function. But for handling regression problem, introducing penalty function containing slack vector is more effective. Therefore, we can get the following formula.

$$
\begin{gathered}
\min \theta=\frac{1}{2}\|w\|^{2}+C \sum_{i=1}^{l}\left(\xi_{i}+\xi_{i}^{*}\right) \\
\text { s.t. }\left\{\begin{array}{c}
y_{i}-\left\langle w, x_{i}\right\rangle-b \leq \varepsilon+\xi_{i} \\
\left\langle w, x_{i}\right\rangle+b-y_{i} \leq \varepsilon+\xi_{i}^{*} \\
\xi_{i}, \xi_{i}^{*} \geq 0
\end{array}\right.
\end{gathered}
$$

If $\mathrm{C}$ is bigger than zero, it is acceptable that $\mathrm{f}(\mathrm{x})$ is slightly greater than $\varepsilon$, which also can be seen as solving a penalty fucniton not sensitive to $\varepsilon$.

$$
|\xi|_{\varepsilon}=\left\{\begin{array}{cc}
0 & \text { if }|\xi| \leq \varepsilon \\
|\xi|-\varepsilon & \text { otherwise }
\end{array}\right.
$$

Only if the sample points in the outside of shaped area, can the penalty funciton be influenced. Slack variable factor $\xi$ represents a penalty factor when sample deviates from the linear function. Similar to SVM classifier, through multiple parameter optimization, we can find a set of optimal parameters of $\gamma, \mathrm{C}$ and $\xi$, making the solution optimal.

\section{The Empirical Analysis}

\subsection{Image Database}

The photo gallery used in existing research is general from internet photo sharing communities' mass photos, such as photo.net, DPChallenge.com, Flickr.com and other famous sites. The existing research provides a lot picture database for the public to choose. We found that the photo database of Datta is the most suitable to our request, therefore adopting image database. We totally download 3467 computer images from Datta, each computer image will be rated by more than ten users, scores range from 1 to 7 points. High space means that the user thinks it good-looking. Referred to Datta's research, we consider the computer images with scores higher than 5.8 as high aesthetic feeling (beauty), computer images with scores lower than 4.2 as low aesthetic (non-beauty), and others are not considered. So far, we have 644 "beauty" pictures, and 750 "non-beauty”, totally 1394 images.

\subsection{The Experimental Results}

The whole experimental process is proceeded in the environment of Window 7 system and $\mathrm{C}++$. In the development process, OpenCV2.3 and Libsvm are used. In addition, the experimental analysis is run on Weka software.

When constructing computer image aesthetic classification model, we use RBF kernel SVM classifier to determine the computer image aesthetics. When training SVM classifier, we also run the multi-parameter optimization steps, we use grid research for multi-parameter optimization.

Table 1 shows the classification test results of SVM classifier, which is a confusion matrix to evaluate classification results. The matrix columns show the predicted classification results, matrix transverse show the real classification results. Confusion matrix average accuracy is the proportion of real samples to total samples, so the classifier average accuracy is $77.42 \%$. 
Table1 Confusion matrix of computer image aesthetics classification model based on SVM.

\begin{tabular}{|c|c|c|}
\hline & \multicolumn{2}{|c|}{ SVM classifier } \\
\hline & Beauty & Non-beauty \\
\hline Beauty & $75.73 \%$ & $19.59 \%$ \\
\hline Non-beauty & $24.68 \%$ & $80.04 \%$ \\
\hline The average accuracy & & \\
\hline
\end{tabular}

The regression analysis is based on SVR algorithm. Table2 shows some indexes commonly used in regression performance evaluation, and compare the paper's method with Datta. Among them, the correlation between the paper's method to computer image aesthetic is 0.787 , while the Datta's is 0.689. To the error indicators, the paper's method has smaller error.

Table 2 Regression performance comparison

\begin{tabular}{ccccc}
\hline & Correlation & Mean absolute error & Average relative error & Residual standard deviation \\
\hline Datta's & 0.689 & 0.535 & 0.103 & 0.618 \\
The paper's & 0.787 & 0.357 & 0.093 & 0.562 \\
\hline
\end{tabular}

\subsection{Feature Analysis}

In order to in-depth analyze the influence of characteristics to computer image aesthetic evaluation, we make the feature selection of SVM classifier. The study found that texture characteristic has larger influence, and the regional characteristic also account for a considerable proportion, which show that regional characteristics can remedy the shortcomings of global characteristics, and improve the overall performance of the classifier. In addition, some new characteristics put forward in this paper, such as dark channel characteristics and color template can help us describe the computer image's aesthetic more accurately and comprehensively. Combing with the advantages of these features, the effect of computer image aesthetic classification model is more ideal.

\section{Summary}

This paper mainly introduced the construction of the computer image aesthetic evaluation model. The characteristics of each computer image were extracted, including low-level visual features and high-level aesthetic characteristics. According to different problems, the paper constructed the computer image aesthetic classification model and prediction model, which were completed respectively by SVM classifier and SVR regression algorithm. The experiment proves that the performance of this method is more effective than existing method.

\section{References}

[1] DATTA R, JOSHI D, LI J et al. Studying Aesthetics in Photographic Images Using a Computational Approach [J]. LEONARDIS A, BISCHOF H, PINZ A. Computer Vision-ECCV 2006, 3953:288-301.

[2] WONG L-K, LOW K-L. Saliency-enhanced image aesthetics class prediction[C]//2009 16th IEEE Inthernational Conference on Image Processing (ICIP). 2009:997-1000.

[3] MACHADO P, CARDOSO A, Computing Aesthetics[C]//Proceeding of the Brazilian Symposium on Artificial Intelligence, SBIA’98. Springer-Verlag, 1998:219-229.

[4] LUO Y. TANG X. Photo and Video Quality Evaluation: Focusing on the Subject[C]//Proceeding of the 10th European Conference on Computer Vision: Part III. Berlin, Heidelberg: Springer-Verlag,2008:386-399.

[5] L I, C K. Computational modeling of visual attention [J]. Nature Revies. Neuroscience, 2001,2(3):194-203. 
[6] Dong Cai. Research on Computational Image Aesthetic Evaluation and Optimizing Composition. South China University of Technology, 2014.

[7] Jingjian Yi. Research on Computational Aesthetic of Image Classification and Evaluation System. South China University of Technology, 2013. 